\title{
Installation and Commissioning of the Helium Refrigeration System for the CMS Magnet
}

\author{
G. Perinić, T. Dupont, D. Legrand, and M. Pezzetti
}

\begin{abstract}
A new helium refrigeration plant with a cooling capacity of $800 \mathrm{~W}$ at $4.45 \mathrm{~K}, 4500 \mathrm{~W}$ between $60 \mathrm{~K}$ and $80 \mathrm{~K}$, and $4 \mathrm{~g} / \mathrm{s}$ liquefaction simultaneously has been installed in its temporary position inside the Compact Muon Solenoid assembly hall at CERN. The commissioning of the compressor station has been achieved, the commissioning of the cold box and the cryostats is under way. First operation results are presented.
\end{abstract}

Index Terms-Compact Muon Solenoid, cryogenics, helium, installation, refrigerator, testing.

\section{INTRODUCTION}

$\mathbf{T}$ HE CMS experiment (Compact Muon Solenoid) is a general-purpose proton-proton detector designed to run at the highest luminosity at the LHC [1]. Distinctive features of the CMS detector include a high-magnetic-field solenoid (4 T) coupled with a multilayer muon system, a fully active scintillatingcrystal electromagnetic calorimeter, a tile hadronic calorimeter, and a powerful inner tracking system.

The $220 \mathrm{t}$ cold mass of the high-magnetic-field solenoid will be indirectly cooled by the means of a thermosiphon system. The required cooling power shall be provided by a dedicated helium refrigeration system which has by now been installed in a temporary position inside the CMS assembly hall. After the commissioning and the fine tuning by the means of a test cryostat, the helium refrigeration system will be connected to the CMS solenoid in order to allow the full cryogenic testing of the solenoid. Upon completion of the surface tests of the solenoid, the cold box will be moved to its final position in the service cavern adjacent to the CMS experimental cavern.

\section{Plant Overview}

The helium refrigeration system is described in [2]. Its refrigeration cycle is based on a modified Claude cycle and provides in steady state a cooling capacity of $800 \mathrm{~W}$ at $4.45 \mathrm{~K}, 4500$ $\mathrm{W}$ between $60 \mathrm{~K}$ and $80 \mathrm{~K}$, and $4 \mathrm{~g} / \mathrm{s}$ liquefaction simultaneously. For the cool down the refrigeration power is boosted by the means of a $\mathrm{LN}_{2}$ pre-cooler. The helium refrigeration system breaks down into three major process units: the compressor station, the cold box and the intermediate cryostat. These units are complemented by a test cryostat which is used during the commissioning for the refrigeration power measurements and for the simulation of the thermal loads in order to allow pre-testing of

Manuscript received October 21, 2003.

The authors are with the European Organization for Nuclear Research (CERN), CH-1211 Geneva 23, Switzerland (e-mail: Goran.Perinic@cern.ch; Thierry.Dupont@cern.ch; Dominique.Legrand@ cern.ch; Marco.Pezzetti@cern.ch).

Digital Object Identifier 10.1109/TASC.2004.831048

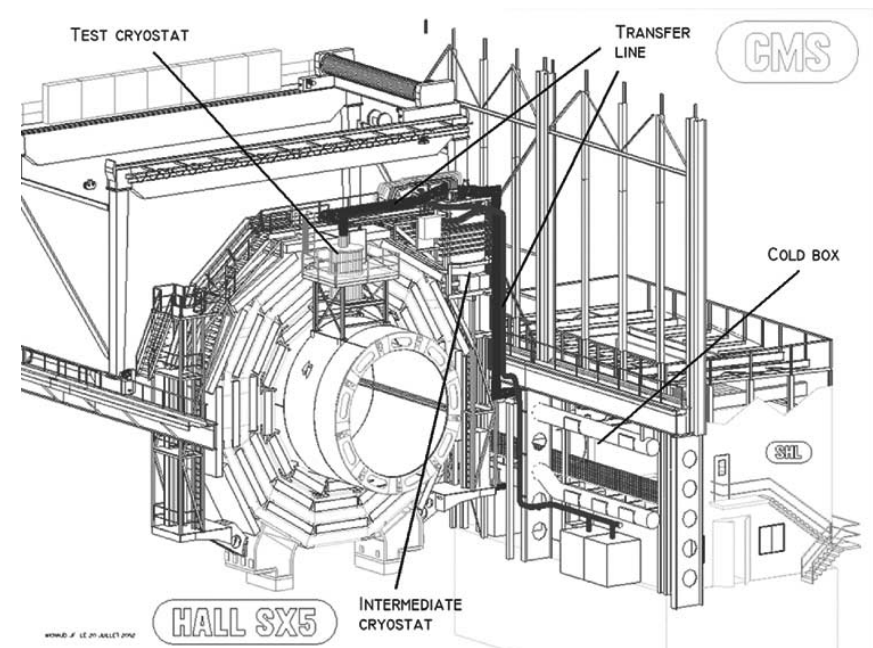

Fig. 1. Commissioning configuration of helium refrigeration plant in the CMS assembly hall.

all possible operation modes. The commissioning configuration is shown in Fig. 1.

All components, but the helium storage tanks and the control system have been supplied by Air Liquide (Sassenage, France). Both, the gaseous helium storage tanks as well as the control system have been procured in the framework of CERN wide contracts.

\section{INSTALLATION}

The installation of the helium refrigeration plant components has been achieved in the period from Apr. 2001 until Sep. 2003.

\section{A. Infrastructure}

The compressor hall and the assembly hall alcove have been purpose built and special requirements like sound proofing and oil retention provisions in the compressor hall and other installation particularities have been implemented. The buildings as well as all services, i.e., the ventilation, the crane, the water cooling circuit, the electrical power supplies, the instrument air compressor etc. have been handed over well in time by the CERN technical services.

\section{B. Compressor Station}

The compressor station has been delivered pre-assembled on four skids (Fig. 2). The first and second stage compressor skids carry the screw compressor, the electric motor and the oil injection circuits. Each of these skids is mounted onto a $15 \mathrm{t}$ concrete block which is decoupled from the ground by the means of 


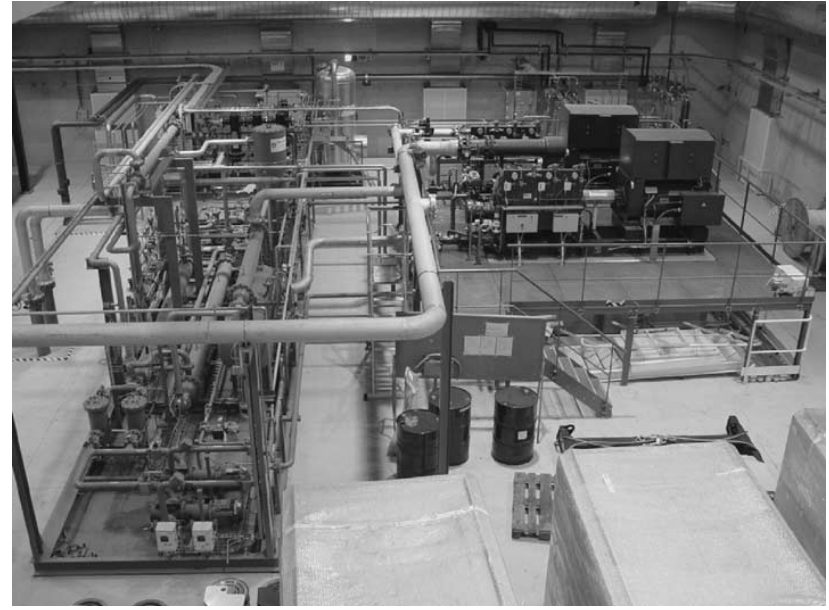

Fig. 2. The compressor station.

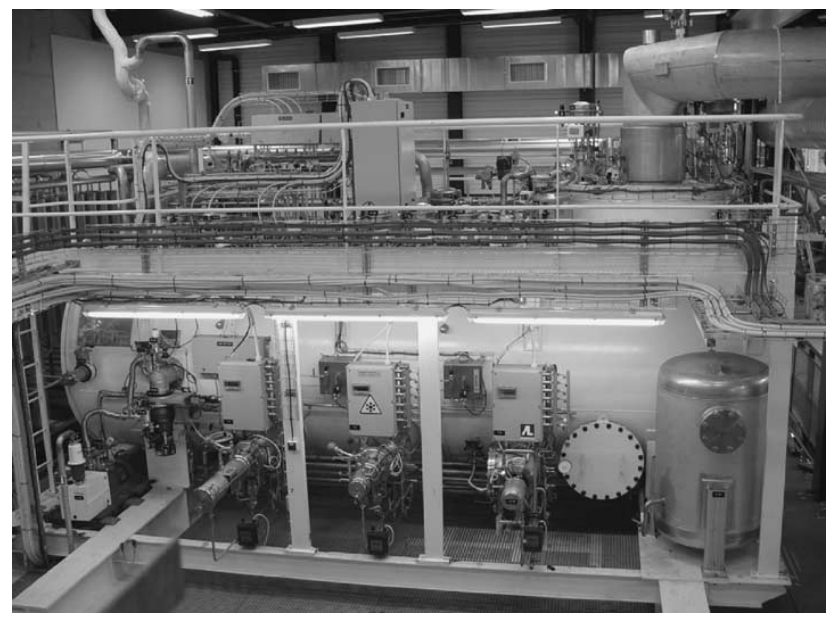

Fig. 3. Cold box in temporary position inside assembly hall.

anti-vibration pads. Access to the two compressor skids at $1.4 \mathrm{~m}$ height is provided by a common platform.

All the remaining components of the compressor station are assembled on two further skids. One skid regroups the primary oil removal, the helium coolers, the oil coolers as well as the doubled oil pumps and oil filters for both compressor stages. The other skid carries the final oil removal train comprising three coalescers, one adsorber and one dust filter, as well as the compressor station pressure controls and the helium analyzer station.

The two $250 \mathrm{~m}^{3}$ helium storage tanks, which have been supplied through an LHC-cryogenics wide contract, had already been commissioned by the mid of 2001 .

\section{Cold Box}

For the surface tests, the cold box has been installed in a temporary position inside an alcove of the assembly hall (Fig. 3).

The cold box as well as its valve panel and the two atmospheric heaters have been installed by lowering them through an opening in the building roof, specially foreseen for this operation. Once all elements were in place, the piping and cabling has been terminated and the cryogenic valve stems and actuators as well as the three turbines have been installed.

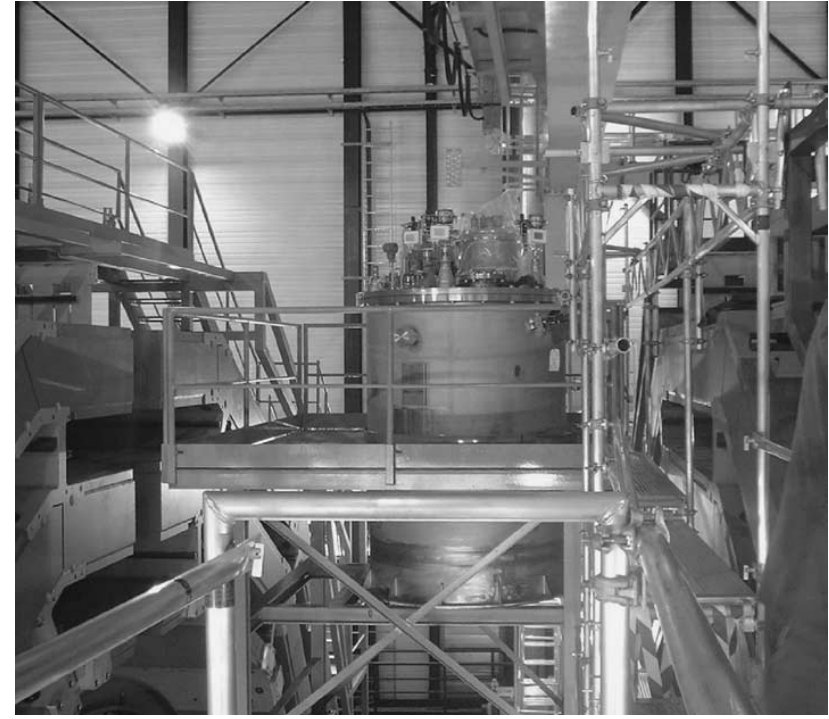

Fig. 4. Test cryostat located on top of the magnet vacuum vessel.

\section{Cryostats}

A number of problems during manufacturing and installation of the intermediate cryostat have led to an important delay with respect to the original planning. At first, due to the problems with the sub-contracted manufacturing, the assembly of the intermediate cryostat had to be terminated in Air Liquide's workshop. Later an unfortunate damage to a cryogenic valve, which has occurred during the installation of the cryostat, required a repair of the cryostat in Air Liquide's workshop and has added an additional 6 month delay. Fortunately these delays could be accommodated without consequences due to the early start of the refrigeration system contract.

The test cryostat (Fig. 4) has been installed on top of the cryostat vacuum vessel near the future position of the magnet phase separator. It allows therefore a test of the refrigeration system close to the operation configuration, i.e., including the intermediate cryostat and the transfer line losses.

\section{E. Controls}

The controls hardware and software has been delivered in three batches, one for the compressor station, one for the cold box and one for the cryostats. Each batch of the controls hardware comprises the remote I/O cubicle, the power supply cubicle and the PLC cubicle. The remote I/O cubicles have been installed adjacent to the corresponding electrical cubicles.

In the course of the project it was decided to order only the software for the compressor station and the cold box via the CERN contract. The software part for the cryostats has been programed in house.

\section{COMMISSIONING}

The commissioning of the helium refrigeration system is carried out in three stages. The compressor station has been commissioned in June and July 2002, the cold box commissioning has started in July 2003 and finally the intermediate and test cryostat commissioning has started in September 2003. 
TABLE I

ISOTHERMAL COMPRESSOR EFFICIENCY

\begin{tabular}{lcc}
\hline \hline & WLV 321 & WLV 204 \\
\hline Inlet pressure & $1 \mathrm{bar}$ & $4.4 \mathrm{bar}$ \\
Inlet temperature & $31.9^{\circ} \mathrm{C}$ & $24.6^{\circ} \mathrm{C}$ \\
Outlet pressure & $4.4 \mathrm{bar}$ & $18.5 \mathrm{bar}$ \\
Mass flow & $210 \mathrm{~g} / \mathrm{s}$ & $210 \mathrm{~g} / \mathrm{s}$ \\
Motor input power & $360 \mathrm{~kW}$ & $344 \mathrm{~kW}$ \\
Overall efficiency $_{\text {Compressor efficiency }}{ }^{\text {a }}$ & $54.6 \%$ & $54.4 \%$ \\
\hline \hline
\end{tabular}

Within each stage, the commissioning has followed the same principle: after installation of both, the plant hardware and the controls hardware, the plant hardware is conditioned. The conditioning comprises drying, purging and evacuation of the helium circuits and filling in of all process fluids. In parallel, a synchronization test of the I/O signals is carried out to ensure the correct communication in between field objects and the plant software. At this stage also the well functioning of every field device is checked, this comprises checks of the range, the function, etc. With all signals in order, the software testing would be tackled by first operating each software module independently and then by running the sequencer automatically. Commissioning is then completed by carrying out the performance tests to verify the plant performance.

\section{A. Compressor Station}

The commissioning of the compressor station could be achieved without problems. The result of the efficiency measurements is listed in Table I. However, after about 500 hours of operation a problem with the oil removal has become apparent as an excessive amount of oil was detected in the last coalescer.

\section{B. Cold Box and Cryostats}

The commissioning of the cold box and the cryostats together with the transfer line is still under way. Up to now a cool down of the cryostats and the transfer line down to below $10 \mathrm{~K}$ and a cool down of the cold box down to liquid helium temperature has been achieved. Full commissioning of the cold box has not yet been possible due to the fact that the third turbine would not start and had to be repaired. We have also encountered some difficulties with the automatic controls of the turbines 1 and 2 which are still under investigation.

\section{Controls}

The process software which is running on two PLCs is working reliably. Only adaptations that resulted from the commissioning had to be implemented. The supervisory software which serves as operator interface and which deals with the data storage and the alarm tasks, however, has been accompanied by numerous teething troubles. This is due to the fact that this software package has been discontinued at CERN shortly after its introduction and we are one of the very few users. We expect these problems to disappear with the replacement of the supervisory software by the new CERN cryogenics wide supervisory system.

\section{OUTLOOK}

After termination of the commissioning, we shall continue with the fine tuning of the helium refrigeration system until the magnet installation will require the cutting of the transfer line and the removal of the test cryostat. The next steps will then be the connection to the magnet phase separator and the cool down and operation of the magnet for the surface tests. All but the compressor station will be moved together with the magnet in 2006 to their final position in the caverns $90 \mathrm{~m}$ underground.

\section{REFERENCES}

[1] A. Herve et al., "Status of the Construction of the CMS Superconducting Magnet at LHC," this conference.

[2] G. Perinić et al., "The helium cryogenic plant for the CMS superconducting magnet," Adv. Cryog. Eng., vol. 47, pp. 232-238, 2002. 